\title{
Mycena sect. Galactopoda: two new species, a key to the known species and a note on the circumscription of the section
}

\author{
Aravindakshan DM and Manimohan P*
}

Department of Botany, University of Calicut, Kerala, 673 635, India

Aravindakshan DM, Manimohan P 2013 - Mycena sect. Galactopoda: two new species, a key to the known species and a note on the circumscription of the section. Mycosphere 4(4), 653-659, Doi 10.5943/mycosphere/4/4/1

\begin{abstract}
Mycena lohitha sp. nov. and M. babruka sp. nov. are described from Kerala State, India and are assigned to sect. Galactopoda. Comprehensive descriptions, photographs, and comparisons with phenetically similar species are provided. A key is provided that differentiates all known species of the sect. Galactopoda. The circumscription of the section needs to be expanded to include some of the species presently assigned to it including the new species described here and a provisional, expanded circumscription of the section is followed in this paper.
\end{abstract}

Key words - Agaricales - Basidiomycota - biodiversity - Mycenaceae - taxonomy

\section{Introduction}

Section Galactopoda (Earle) Maas Geest. of the genus Mycena (Pers.) Roussel (Mycenaceae, Agaricales, Basidiomycota) comprises species with medium-sized basidiomata with stipes that often exude a fluid when cut, exhibit coarse whitish fibrils at the base, and turn blackish when dried. Additionally they have ellipsoid and amyloid basidiospores, cheilocystidia that are generally fusiform and often with coloured contents, and hyphae of the pileipellis and stipitipellis covered with excrescences and diverticulate side branches. Most of these species have been recorded as growing on decaying wood. Maas Geesteranus (1988) broke apart the former section Lactipedes (that comprised species exuding a milky or coloured latex when cut) into four: sect. Lactipedes, sect. Sanguinolentae, sect. Galactopoda, and sect. Crocatae. Maas Geesteranus (1988), when he gave sectional status to Galactopoda, included only M. haematopus (Pers.) P. Kumm. in that section. Mycena haematopus is a widely distributed species known from Europe, the United States, Canada, Japan, Australia and Argentina. In addition to M. haematopus, the section at present incorporates three Australian species (Mycena toyerlaricola Grgur., M. kuurkacea Grgur. and M. insueta Grgur.), three New Zealand species (M. mariae G. Stev., M. ura Segedin and M. parsonsiae G. Stev.) and a Brazilian species (M. rubrofarcta Maas Geest. \& de Meijer) (Segedin 1991, Maas Geesteranus \& de Meijer 1997, Grgurinovic 2002). During the course of our studies on mycenas of Kerala State, India, we came across two hitherto undescribed species that we consider as belonging in this section and they are described and discussed here.

\section{Methods}

Conventional morphology-based taxonomic methods were employed for this study. 
Microscopic observations were made on material stained with $1 \%$ aqueous solution of Congo red and mounted in 3\% aqueous $\mathrm{KOH}$. Melzer's reagent was used to observe whether the spores and tissues were amyloid. For evaluation of the range of spore-size, twenty basidiospores each from one specimen of each collection cited were measured. Colour codes used in the descriptions are from Kornerup \& Wanscher (1978). All examined collections cited, including the holotypes, are deposited at the Kew (Mycology) herbarium and their Kew accession numbers (e.g., K(M) 178337) are provided. Maas Geesteranus' $(1988,1992)$ concept of Mycena sect. Galactopoda has been slightly expanded as discussed later in this paper.

\section{Results and Discussion}

\section{Taxonomy}

Mycena lohitha Aravind. \& Manim., sp. nov. MycoBank MB 803910

Fig. $1 \mathrm{a}-\mathrm{g}$

Characerised by small reddish basidiomata that turn blackish when dried, pinkish white lamellae with dark brown edges and dark brown dotted sides, dimorphic cheilocystidia with coloured contents, fusiform pleurocystidia with coloured contents, ellipsoid and amyloid basidiospores, slightly gelatinized, nodulose-diverticulate pileipellis hyphae and stipitipellis hyphae that are smooth in most parts of the stipe. Differing from Mycena toyerlaricola and M. kuurkacea in having somewhat smaller basidiomata, lamellae that are punctate with reddish brown dots and slightly gelatinized pileipellis and stipitipellis hyphae with coloured contents.

Etymology - lohitha (Sanskrit), reddish.

Basidiomata small. Pileus $4-8 \mathrm{~mm}$ diam., up to $5 \mathrm{~mm}$ high, conic when young, becoming conico-campanulate with age; surface brownish red (9D8) at the centre and scarlet (9A8) elsewhere when fresh, soon changing to dark brown $(8 \mathrm{~F} 8)$ at the centre as well as on the striations and margin and brown (8E6) or reddish brown (8D5) elsewhere, scrobiculate on disc, slightly sulcate at middle, translucent-striate, glabrous, dry; margin incurved and entire when young, becoming straight and undulate with age. Lamellae $12-14$ reaching the stipe, adnate, pinkish white, up to $0.75 \mathrm{~mm}$ wide, subdistant, with lamellulae of 1-3 lengths; sides of the lamellae punctate with dark brown dots; edge finely hairy or eroded under a lens, dark brown (8F8). Stipe 16-33 × 1-1.5 mm, central, terete, slightly tapering towards apex, hollow; surface brownish red (9D8) to dark brown (8F4) towards apex, reddish white (8A2) towards base, pruinose at apex, glabrous towards base; base swollen, with strigose basal mycelium. Context not conspicuous, less than $0.5 \mathrm{~mm}$ wide, concolorous with the pileus. Odour and taste not recorded. Basidiomata turns blackish when dry.

Basidiospores 8.5-11 × 4-5.5 (9.7 $\pm 0.637 \times 4.65 \pm 0.462) \mu \mathrm{m}, \mathrm{Q}=1.73-2.38, \mathrm{Qm}=2.1$, ellipsoid, thin-walled, hyaline, smooth, amyloid, with guttules. Basidia 20-29 $\times 8-9.5 \mu \mathrm{m}$, clavate, bearing 4 sterigmata up to $7 \mu \mathrm{m}$ long. Cheilocystidia crowded, of two types: one type 31-70 $\times 7.5-$ $14 \mu \mathrm{m}$, narrowly or broadly fusiform with acute or acuminate or mucronate apex, thin-walled, with greyish contents, smooth; other type 6-36 $\times 3-11 \mu \mathrm{m}$, clavate to cylindrical, thin-walled, with greyish contents, and with somewhat branched, straight or curved excrescences $(2-18.5 \times 1-4 \mu \mathrm{m})$. Pleurocystidia 26.5-53 $\times 10-14 \mu \mathrm{m}$, scattered, narrowly or broadly fusiform, thin-walled, smooth, hyaline or with brownish contents. Lamellar trama subregular; hyphae 2.5-16 $\mu \mathrm{m}$ wide, thinwalled, hyaline, vinoid in Melzer's reagent. Subhymenium ramose. Pileus trama subregular; upper hyphae $6.5-25 \mu \mathrm{m}$ wide, thin- to slightly $(0.5-0.75 \mu \mathrm{m})$ thick-walled, with reddish to blackish contents; lower hyphae 1.5-15 $\mu \mathrm{m}$ wide, thin-walled, hyaline, vinoid in Melzer's reagent. Pileipellis an epicutis; hyphae 1-4.5 $\mu \mathrm{m}$ wide, thin-walled, with dark grey or reddish or blackish grey contents, with simple or furcate, straight, curved or flexuous excrescences $(0.5-13 \times 1-2 \mu \mathrm{m})$, slightly gelatinized. Stipitipellis a cutis; hyphae $2-6 \mu \mathrm{m}$ wide, slightly gelatinized, thin-walled, with dark grey or reddish or blackish grey contents, smooth in most parts of the stipe but nodulosediverticulate $(0.5-6 \times 1-4 \mu \mathrm{m})$ at the stipe apex. Caulocystidia absent. Stipe trama dextrinoid to vinoid in Melzer's reagent. Clamp connections seen on all hyphae. 


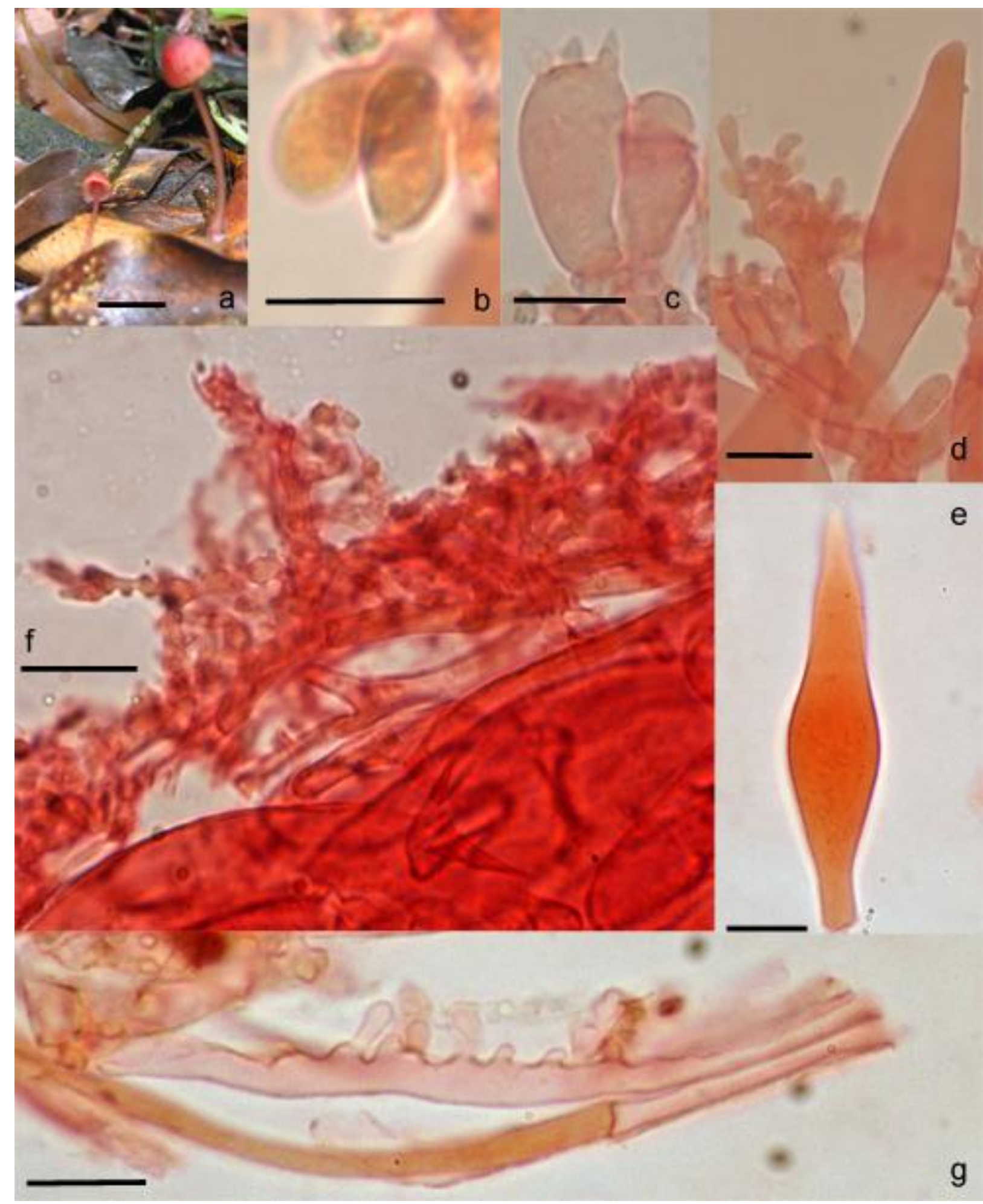

Fig. 1 a-g - Mycena lohitha. a Basidiomata b Basidiospore. c Basidium. d Cheilocystidia. e Pleurocystidium. f Pileipellis. g Stipitipellis. - Bars $=10 \mathrm{~mm}$ for basidiomata and $10 \mu \mathrm{m}$ for microstructures.

On decaying leaves, scattered, June.

Distribution - known only from the type locality.

Material examined - India, Kerala State, Wayanad District, Meppadi, Thollayiram Forest, 21 June 2007, D.M. Aravindakshan DM135 (K(M) 178337, holotype).

Small reddish basidiomata that turn blackish when dried, pinkish white lamellae with dark brown edges and dark brown dotted sides, dimorphic cheilocystidia with coloured contents, 
fusiform pleurocystidia with coloured contents, ellipsoid and amyloid basidiospores, slightly gelatinized, nodulose-diverticulate pileipellis hyphae and stipitipellis hyphae that are smooth in most parts of the stipe are the diagnostic features of this species. Many of these characters are indicative of the sect. Galactopoda and hence this species is placed in that section although the bleeding nature of its stipe, a key feature of members of the section, remains to be verified.

Mycena haematopus differs from the present species in having larger basidiomata, densely pruinose pileus and stipe surfaces, pinkish brown or pale purplish brown pileus, numerous lamellae (15-20) which are broader (up to $3.5 \mathrm{~mm}$ ), larger basidia, non-gelatinized pileipellis and stipitipellis, and caulocystidia. Mycena toyerlaricola and M. kuurkacea show striking similarity to the present species in having brownish red or brick red coloured pileus, coloured lamella-edge, two types of cheilocystidia and coloured contents in cheilocystidia, pileocystidia and pileus trama. But those species differ from $M$. lohitha in having somewhat larger basidiomata, lamellae that are not punctate with reddish brown dots and non-gelatinized pileipellis and stipitipellis hyphae with coloured contents. Mycena insueta differs in having larger basidiomata, brownish orange to greyish orange pileus, numerous lamellae (c. 20) that are without reddish brown-dotted sides, subglobose basidiospores, larger basidia, much larger cheilocystidia, infrequent pleurocystidia, pseudocystidia and caulocystidia and non-gelatinized pileipellis. Mycena mariae differs in having pseudocystidia, subglobose basidiospores $(\mathrm{Q}=1.4)$ and laticiferous hyphae in the hymenophoral trama. Mycena ura differs in having larger basidia, cheilocystidia and pleurocystidia, cystidioid pileipellis hyphal ends, and red strigose hairs on the stipe. Mycena parsonsiae differs in having subglobose basidiospores, characteristically short and inflated basidia, exclusively fusoid cheilocystidia, distinct caulocystidia and non-gelatinized hyphae of the pileipellis. Mycena rubrofarcta differs in having somewhat larger basidiomata, concolorous lamella-edge, smaller basidiospores $(6.5-7.2 \times$ 4.8-5.4 $\mu \mathrm{m}$ ), long-stalked, narrowly fusiform cheilocystidia with their tips surrounded by a globose mass of exudates, and non-gelatinized pileipellis.

Mycena babruka Aravind. \& Manim., sp. nov. MycoBank MB 803912

Fig. $2 \mathrm{a}-\mathrm{g}$

Characterized by small basidiomata that turn blackish on drying, dark brown pileus, decurrent lamellae with reddish brown lamella-edge, mild alkaline odour, bitter taste, stipe that exudes a fluid when cut, ellipsoid and amyloid basidiospores, cheilocystidia with dark contents and with excrescences at the apex, slightly gelatinized and diverticulate pileipellis hyphae and absence of pleurocystidia. Differing from all other species of sect. Galactopoda in having a combination of features such as dark brown pileus, decurrent lamellae, absence of fusoid cheilocystidia and slight gelatinization of the pileipellis hyphae.

Etymology - babruka (Sanskrit), brownish.

Basidiomata small. Pileus 4.5-11 mm diam., 2-5 mm high, convex with a very slight central depression when young, becoming broadly convex or nearly applanate still with the central depression; surface dark brown (8F8) at the centre and on the striations and greyish orange (5B3) towards margin when young, becoming dark brown (7F8) at the centre and on the striations and light brown (6D4) or brownish orange (6C4) towards margin and brown (7E6) on margin with age, translucent- to sulcate-striate towards margin, glabrous; margin straight and entire when young, becoming upturned and undulating with age. Lamellae 11-15 reaching the stipe, decurrent to deeply decurrent, pinkish white (7A2) or reddish grey (7B2), thin, 1-1.5 mm wide, subdistant, with lamellulae of 1-3 lengths; edge finely pruinose under a lens, initially brown (7E6) or dark brown (7F8) or reddish brown (8F8) where the lamella meets the stipe and the pileus margin, gradually entire edge becoming coloured. Stipe $15-58 \times 0.5-1 \mathrm{~mm}$, central, terete, almost equal, hollow; surface translucent, brown (7E6) at the apex and on the base, yellowish brown (5D4) at the middle when young, becoming light brown (6D5 or 6D4) at base, reddish blonde (5C4) or greyish orange (5B3) elsewhere with age, glabrous; base slightly broad, with white, hispid basal mycelium. Context not conspicuous. Odour slightly alkaline. Taste bitter. Exuding a dark brown (7F8, 8F8) fluid when the stipe is cut. Basidiomata turns blackish when dry. 


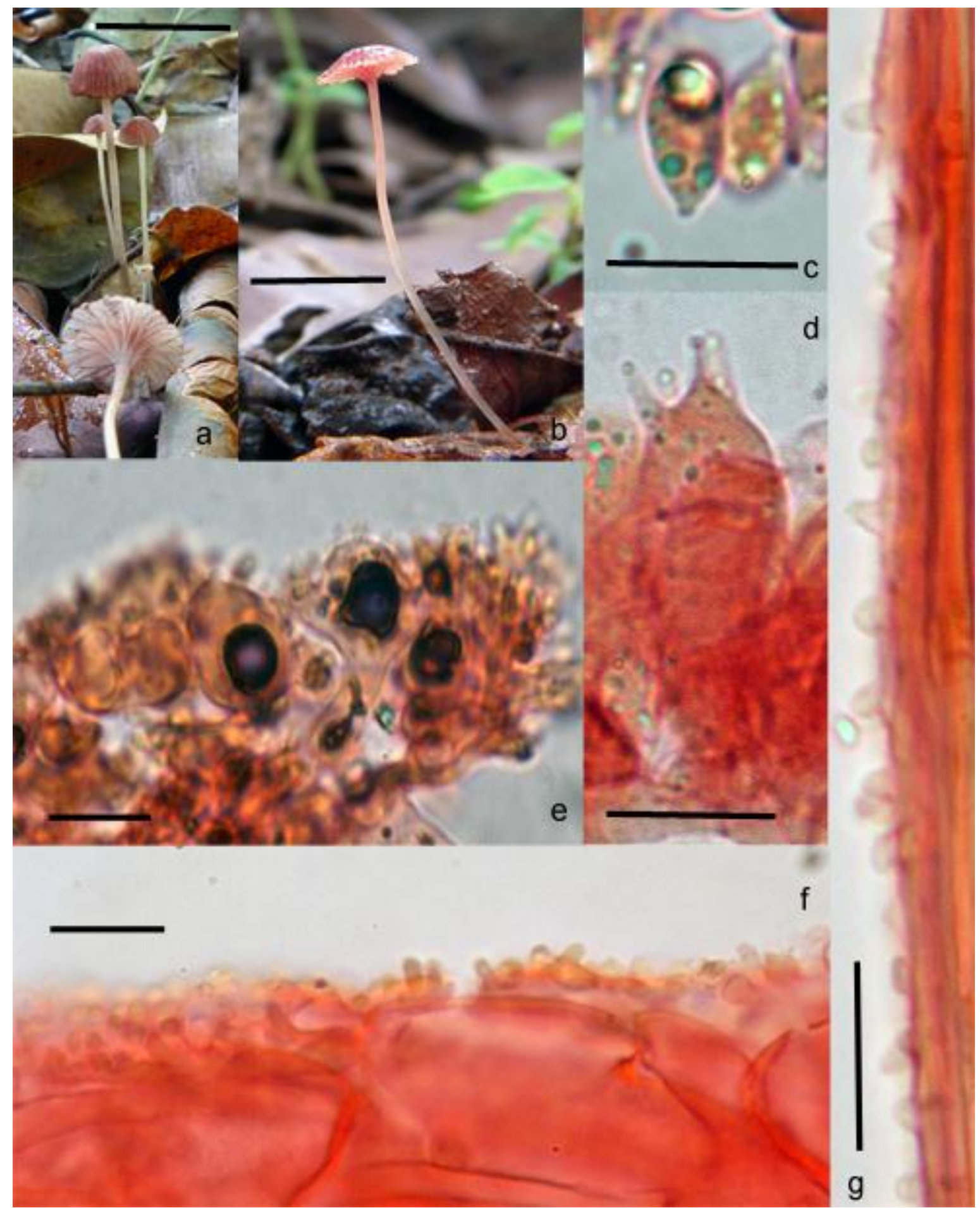

Fig. 2 a-g - Mycena babruka. a, b Basidiomata. c Basidiospore. d Basidium. e Cheilocystidia. f Pileipellis. g Stipitipellis. - Bars $=10 \mathrm{~mm}$ for basidiomata and $10 \mu \mathrm{m}$ for micro-structures.

Basidiospores 6-8.5 $(9.5) \times 3-4(7.5 \pm 0.646 \times 3.769 \pm 0.307) \mu \mathrm{m}, \mathrm{Q}=1.63-2.83, \mathrm{Qm}=2$, ellipsoid, thin-walled, hyaline, smooth, amyloid, with refractive guttules. Basidia 17-24 × 6-7.5 $\mu \mathrm{m}$, clavate, bearing 4 sterigmata up to $7 \mu \mathrm{m}$ long. Cheilocystidia $12-28(44) \times 5-16 \mu \mathrm{m}$, clavate or broadly clavate or irregularly-shaped, thin-walled, with dark brownish-red to blackish contents, with simple or branched coarse excrescences $(1-6(13.5) \times 1-3 \mu \mathrm{m})$ at the top. Pleurocystidia none. Lamellar trama subregular; hyphae 2-19 $\mu \mathrm{m}$ wide, thin-walled, hyaline, vinoid in Melzer's reagent. Subhymenium ramose. Pileus trama subregular; hyphae 6-34 $\mu \mathrm{m}$ wide, thin-walled, hyaline, vinoid in Melzer's reagent. Pileipellis a cutis; hyphae 1-4 $\mu \mathrm{m}$, slightly gelatinized, thin-walled, with 
reddish or reddish-brown contents, with simple or branched digitate excrescences (1-7 (12) $\times$ 1-2.5 $\mu \mathrm{m})$. Stipitipellis a cutis; hyphae 1.5-7 $\mu \mathrm{m}$ wide, thin-walled, hyaline to pale brownish, with simple excrescences $(2-8 \times 2-3 \mu \mathrm{m})$ more towards the stipe apex. Stipe trama dark brown in water, vinoid in Melzer's reagent. Clamp connections seen on all hyphae.

On decaying leaves and twigs, scattered, June.

Distribution - known only from two localities in Kerala State, India.

Material examined - India, Kerala State, Malappuram District, Calicut University Campus, 22 June 2009, D.M. Aravindakshan DM253 (K(M)178338, holotype); Kannur District, Neeliyarkotta: 17 June 2009, D.M. Aravindakshan DM260 (K(M)178339).

Small basidiomata that turn blackish on drying, dark brown pileus, decurrent lamellae with reddish brown lamella-edge, mild alkaline odour, bitter taste, stipe that exudes a fluid when cut, ellipsoid and amyloid basidiospores, cheilocystidia with dark contents and with excrescences at the apex, slightly gelatinized and diverticulate pileipellis hyphae and absence of pleurocystidia are the diagnostic features of the present species. Sectional level placement of this species is somewhat difficult. Characters such as ellipsoid and amyloid basidiospores, exudative stipe, non-separable pileipellis and clavate cheilocystidia with cylindrical excrescences may indicate sect. Crocatae Maas Geest. However, that section is further characterized by ascending lamellae with whitish edge and pleurocystidia. Characters such as stipe exuding a dark fluid when cut, bitter taste, blackening basidiomata, coloured lamella-edge, vinescent lamellar trama, and ellipsoid and amyloid basidiospores strongly indicate sect. Galactopoda. Although sect. Galactopoda is further characterized by ascending, adnate lamellae and fusoid cheilocystidia with slender neck, we consider that section as more appropriate for Mycena babruka. Mycena lohitha, described earlier in this paper, differs from $M$. babruka in having reddish pileus, adnate lamellae with sides punctate with dark brown dots, dimorphic cheilocystidia, fusoid pleurocystidia and larger basidiospores. A combination of features such as the dark brown pileus, decurrent lamellae, absence of fusoid cheilocystidia and the slight gelatinization of the pileipellis hyphae makes M. babruka distinct from all other species of sect. Galactopoda.

The following key differentiates all known species of the sect. Galactopoda:

1 Basidiospores subglobose 2

1 Basidiospores ellipsoidal

2 Basidiospores $6.5-9 \times 5.5-7 \mu \mathrm{m}$; pileipellis hyphae without inflated excrescences

Mycena parsonsiae

2 Basidiospores 7-11 ×5.2-7.9 $\mu \mathrm{m}$; pileipellis hyphae with inflated excrescences ... Mycena insueta

3 Lamellae consistently decurrent; pleurocystidia absent; basidiospores 6-8.5 × 3-4 $\mu \mathrm{m}$

Mycena babruka

3 Lamellae not consistently decurrent; pleurocystidia present .............................................. 4

4 Basidiomata growing mostly on decaying litter; caulocystidia absent ....................................... 5

4 Basidiomata growing on decaying wood; caulocystidia present ......................................... 9

5 Cheilocystidia dimorphic, both smooth, fusoid ones as well as clavate elements with cylindrical excrescences at the top.

5 Cheilocystidia monomorphic, fusoid; basidiospores 8.5-11.5 $\times 5.5-7 \mu \mathrm{m}$. Mycena mariae

6 Lamellae punctate with reddish brown dots; hyphae of the pileipellis and the stipitipellis slightly gelatinized; basidiospores $8.5-11 \times 4-5.5 \mu \mathrm{m}$ Mycena lohitha

6 Lamellae not punctate with reddish brown dots; hyphae of the pileipellis and the stipitipellis nongelatinized

7 Base of stipe with red hairs; pileipellis elements associated with conidia-like structures; basidiospores $7.5-10 \times 4.5-5.5 \mu \mathrm{m}$ Mycena ura

7 Base of stipe not with red hairs; pileipellis elements not associated with conidia-like structures... 8 8 Basidiomata dull reddish, pseudocystidia present; basidiospores 7-11 $\times 4.5-7.3 \mu \mathrm{m}$. 
8 Basidiomata bright red; pseudocystidia absent; basidiospores $6.5-10.5 \times 3.2-6.5 \mu \mathrm{m}$

Mycena toyerlaricola

9 Cheilocystidia sessile or short-stalked; basidiospores 9-9.5 $\times 5.3-5.8 \mu$ m........Mycena haematopus

9 Cheilocystidia often long-stalked; basidiospores $6.5-7.2 \times 4.8-5.4 \mu \mathrm{m}$ Mycena rubrofarcta

As noted by Grgurinovic (2002), the circumscription of the section needs to be broadened to include some of the species presently assigned to it. An analysis of the characters of the species currently placed in it including the species described here indicates that characters such as attachment of lamellae, spore shape, morphology of the cheilocystidia and the substratum on which the basidiomata grow have more than one character-state possible in the section. According to the circumscription given by Maas Geesteranus (1988), the section is characterized, apart from other characters, by adnate lamellae, pip-shaped basidiospores, fusiform cheilocystidia and basidiomata that grow on decaying wood. However, some species that were assigned to the section since then have character-states different from those given in the circumscription. For example, Mycena insueta and M. parsonsiae have subglobose spores (Segedin 1991, Grgurinovic 2002). Mycena toyerlaricola (Grgurinovic 2002), M. lohitha and M. babruka are known to grow on leaf litter. Mycena babruka has decurrent lamellae. The most pronounced deviation observed, in our opinion, is in cheilocystidial morphology. Species such as Mycena toyerlaricola, M. kuurkacea (Grgurinovic 2002), M. ura (Segedin 1991) and M. lohitha have, in addition to the typical fusoid ones, another kind with somewhat cylindrical, rarely branched excrescences at the top. In the case of M. babruka, fusoid cheilocystia are entirely replaced by cheilocystidia with branched coarse excrescences at the top. In this paper, we follow a provisional, expanded circumscription of the section that incorporates the aforesaid character states as well.

\section{References}

Grgurinovic CA. 2002 - The Genus Mycena in South-Eastern Australia. Fungal Diversity Press, Hong Kong.

Kornerup A, Wanscher JH. 1978 - Methuen Handbook of Colour. 3rd Ed. Eyre Methuen, London.

Maas Geesteranus RA. 1988 - Conspectus of the Mycenas of the Northern Hemisphere 10. Sections Lactipedes Sanguinolentae Galactopoda and Crocatae. Proceedings of the Koninklijke Nederlandse Akademie van Wetenschappen Series C: Biological and Medical Sciences 91(4), 377-404.

Maas Geesteranus RA. 1992 - Mycenas of the Northern Hemisphere II. Conspectus of the Mycenas of the Northern Hemisphere. North-Holland, Amsterdam.

Maas Geesteranus, RA, de Meijer AAR. 1997 - Mycenae Paranaenses. North-Holland, Amsterdam.

Segedin BP. 1991 - Studies in the Agaricales of New Zealand: some Mycena species in sections Longisetae, Polyadelpha, Rubromarginatae, Galactopoda, Lactipedes, and Calodontes. New Zealand Journal of Botany 29, 43-62. 